\title{
On certain families of planar patterns and fractals
}

\author{
Ligia L. Cristea \\ Karl-Franzens-Universität Graz \\ Institut für Mathematik und Wissenschaftliches Rechnen \\ Heinrichstrasse 36, 8010 Graz, Austria \\ strublistea@gmail.com
}

December 8, 2017

This paper is dedicated to Professor Robert F. Tichy on the occasion of his
$60^{\text {th }}$ anniversary.

\begin{abstract}
This survey article is dedicated to some families of fractals that were introduced and studied during the last decade, more precisely, families of Sierpiński carpets: limit net sets, generalised Sierpiński carpets and labyrinth fractals. We give a unifying approach of these fractals and several of their topological and geometrical properties, by using the framework of planar patterns.
\end{abstract}

Keywords: fractal, Sierpiński carpet, pattern, graph, connectedness, dendrite, path length, arc length

MSC: 28A80, 05C38, 28A75, 51M25, 54D05, 54F50

\section{$1.1 \quad$ Introduction}

Sierpinski carpets are self-similar fractals in the plane that originate from the classical Sierpiński carpet $[27,41]$. Sierpiński carpets are constructed by dividing the unit square

DOI: https://doi.org/10.5592/CO/CCD.2016.01 
On certain families of planar patterns and fractals

into $m \times m$ congruent smaller subsquares of which $m_{0}$ squares are cut out together with their boundary, and then taking the closure. The resulting pattern is the generator of the Sierpiński carpet. At each step of the iterative construction this procedure is applied to all remaining squares, and, repeating this construction ad infinitum, the resulting object is a fractal of Hausdorff dimension $\frac{\log \left(m^{2}-m_{0}\right)}{\log (m)}$, called a Sierpiński carpet [20]. Figure 5 shows the first two steps of the iterative construction of a Sierpiński carpet.

These fractals can also be defined as attractors of IFS (for Iterated Functions Systems we refer, e.g., to the books of Falconer [18, 19] and Barnsley [3, 4]), and occur in several branches of mathematics. In particular, their geometric and topological properties gained a lot of interest, see, e.g., Whyburn [43], Curtis and Fort [16], McMullen [31], Bandt and Mubarak [1], Lau et al. [25]. During the last decades Sierpiński carpets have been used, e.g., as models for porous materials [20, 42].

Limit net sets, generalised Sierpiński carpets and labyrinth fractals are families of Sierpiński carpets that were introduced and studied by Cristea and Steinsky $[8,9,10,12$, $13]$ and some of the results were extended in recent research $[14,15]$ to even more general fractal objects called mixed labyrinth fractals. Studying these objects is of interest not just for mathematics, but also for research in physics, where some of the results have already been used, e.g., [36, 22, 37].

In this paper we present results on topological and geometrical properties that were obtained for the three families of Sierpiński carpets mentioned above, such as connectedness or lengths of arcs in these fractals, everything being done under a combinatorial frame, where the combinatorial character of the problems comes from the combinatorics of the generator(s) of the carpet: the pattern(s).

Although originally net sets and limit net sets were defined and constructed by means of net matrices [8], and the labyrinth fractals by using labyrinth sets [12, 13], throughout this paper we give a unifying approach of all the families of carpets mentioned above by means of patterns, as it was done in the case of the generalised Sierpiński carpets that were studied $[9,10]$ after the other mentioned carpets, and in more recent work, for mixed labyrinth fractals $[14,15]$.

Graph directed constructions, see, e.g., [29], GDMS (Graph Directed Markov Systems, see, e.g., [30]), and random fractals [17, 28] also offer frameworks for studying the objects that occur along this paper. Finally, we mention that there are recent results and ongoing research on $V$-variable fractals, see e.g. [21], and several of the fractals studied and mentioned in this section can be approached within the frame of $V$-variable fractals. For $V$-variable fractals and super-fractals we also refer to Barnsley's book [4].

Let us now give a short outline of the paper. In Section 1.2 we define planar patterns and the graph associated to a planar pattern. Section 1.3 is dedicated to net sets and 
limit net sets. In Section 1.4 we briefly present recent research on generalised Sierpiński carpets. Section 1.5 deals with results about self-similar and mixed labyrinth fractals and also refers to very recent results. Finally, Section 1.6 is dedicated to conclusions and final remarks of the survey.

\subsection{Planar patterns and Sierpiński carpets}

First, let us recall the definition of a pattern, as it is given in some of the above mentioned papers $[9,10]$. Let $x, y, q \in[0,1]$ such that $Q=[x, x+q] \times[y, y+q] \subseteq$ $[0,1] \times[0,1]$. Then for any point $\left(z_{x}, z_{y}\right) \in[0,1] \times[0,1]$ we define the function $P_{Q}\left(z_{x}, z_{y}\right)=$ $\left(q z_{x}+x, q z_{y}+y\right)$.

Let $m \geq 1$. For the integers $i, j$ with $0 \leq i, j \leq m-1$, let $S_{i, j}^{m}=\left\{(x, y) \mid \frac{i}{m} \leq x \leq\right.$ $\frac{i+1}{m}$ and $\left.\frac{j}{m} \leq y \leq \frac{j+1}{m}\right\}$, and $\mathcal{S}_{m}=\left\{S_{i, j}^{m} \mid 0 \leq i \leq m-1\right.$ and $\left.0 \leq j \leq m-1\right\}$. We call any nonempty $\mathcal{A} \subseteq \mathcal{S}_{m}$ an $m \times m$ pattern or, in short, m-pattern.
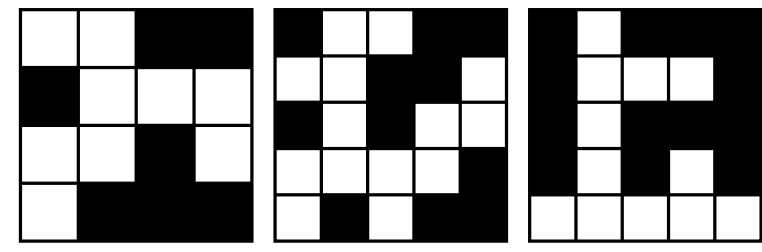

Figure 1: Three patterns, $\mathcal{A}_{1}$ (a 4-pattern), $\mathcal{A}_{2}$ (a 5-pattern) and $\mathcal{A}_{3}$ (a 5 pattern)

In Figure 1 we show three such patterns that are in particular also labyrinth patterns, which we define in Section 1.5. We mention that throughout this paper we think of the black regions in the figures as being "cut out" at the corresponding step, and subsequently the closure (with respect to the topology induced by the Euclidean metric in the plane) of the remainder set is taken.

All families of fractals that we present in this paper can be constructed by means of patterns, and in each case we use an iterative construction, analogous to that described in Section 1.1 for a Sierpiński carpet.

For any pattern $\mathcal{A} \subseteq \mathcal{S}_{m}$, we define the graph $\mathcal{G}(\mathcal{A}) \equiv(\mathcal{V}(\mathcal{G}(\mathcal{A})), \mathcal{E}(\mathcal{G}(\mathcal{A})))$ to be the graph of $\mathcal{A}$, i.e., the graph whose vertices are the (closed) white squares in $\mathcal{A}$, i.e., $\mathcal{V}(\mathcal{G}(\mathcal{A}))=\mathcal{A}$ and whose set of edges $\mathcal{E}(\mathcal{G}(\mathcal{A}))$ consists of the unordered pairs of white squares, that share a common side. 

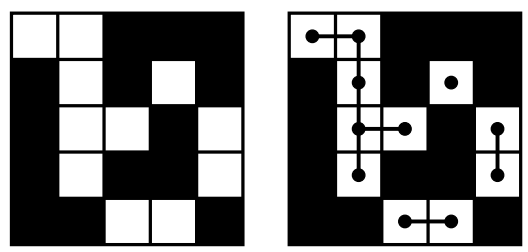

Figure 2: A 5-pattern $\mathcal{A}$ on the left, and the pattern with its corresponding graph $\mathcal{G}(\mathcal{A})$ on the right

\subsection{Limit net sets}

Net set and limit net set are new concepts developed in [8], based, on the one hand, on the observation that various porous materials present holes that at each scale are "evenly" distributed, and, on the other hand, on the distribution properties of $(t, m, s)$ nets, that are well distributed point sets in the unit cube, for more details see [33].

Originally, the net sets were defined [8] with the help of net matrices, that are $4 \times 4$ matrices having all entries from the set $\{0,1\}$. Here we give a (shorter) equivalent definition of the net sets, that is appropriate for the framework of the present paper. We call a $4 \times 4$ pattern a net pattern if each of the four columns and each of the four rows (each containing four squares) contains exactly one black square, and inside each of the four subsquares of side-length $\frac{1}{2}$ of the unit square that share one vertex with the unit square there lies exactly one of the black squares mentioned above. There exist 16 such net patterns, four of them are shown in Figure 3, the other 12 can be obtained by flipping or rotating these. The union of all (closed) white squares in a given net pattern is the corresponding net set of level 0 .
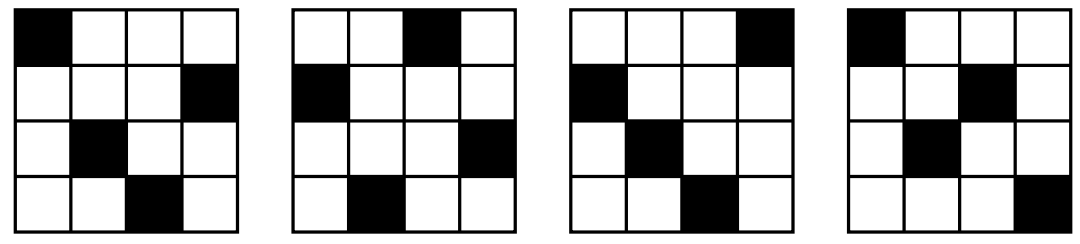

Figure 3: Examples of net patterns

The iterative construction of a sequence of nested sets (net sets of level $1,2, \ldots$ ) is analogous to that described in the introduction of Section 1.1 for the Sierpiński carpets, 
On certain families of planar patterns and fractals

but there is one essential difference: each white square of some level can be replaced, by a so-called net substitution, by any net set of level 0 scaled correspondingly, such that different white squares can be replaced by different net sets.

We call a net substitution uniform if all white squares of a net set of some level are substituted by the same scaled net set. Correspondingly, a net set of some level $k \geq 1$ is uniform if at each step of its construction a uniform net substitution (not necessarily the same) was applied.

Thus, by starting with a net pattern and the corresponding net set $E_{0}$ of level 0 , one obtains, by applying net substitutions, a decreasing sequence of net sets $E_{0} \supset E_{1} \supset$ $\ldots E_{k-1} \supset E_{k} \supset \ldots$

The fractal $E_{\infty}:=\bigcap_{k \geq 0} E_{k}$ obtained as the limit set of this construction is called the limit net set of the sequence $\left\{E_{k}\right\}_{k \geq 0}$ and can be viewed as the limit set of a Moran construction $[32,35,28]$ with Hausdorff and box-counting dimension $1+\frac{\log 3}{\log 4}$.

If at each step of the construction we apply a uniform net substitution, not necessarily the same, then the sets $E_{k}$ of the above sequence are called uniform net sets, and $E_{\infty}$ is called a uniform limit net set. A totally uniform net set is obtained if all substitutions use the same pattern, i.e., we apply the same net substitution at all steps, for all white squares. In this case the obtained limit net set is self-similar: it is a Sierpiński carpet (as defined in the introduction).

When studying connectedness properties of net sets and limit net sets, it is essential to identify two types of patterns: connected and disconnected net patterns. In terms of the graph of the pattern a connected net pattern is a pattern whose graph is connected. Otherwise, the net pattern is called disconnected.

Among other, it was proven that connected net patterns (or, originally, connected net matrices [8]) always produce connected limit net sets. For example, in Figure 3, the first, second and fourth net pattern are connected, while the third is a disconnected net pattern.

Criteria for different "degrees" of connectedness of these fractals have been proven. There are four different possible connectedness "degrees" for limit net sets: netconnectedness (a notion introduced in [8]), connectedness, disconnectedness and total disconnectedness [8]. It was shown how the connectedness or disconnectedness of the net patterns involved in the iterative construction affects the connectedness "degree" of the resulting fractal. Necessary and sufficient conditions for the net-connectedness of the fractal were proven, as well as necessary and sufficient conditions for the total disconnectedness of the fractals, necessary and sufficient conditions for a uniform limit net set to be connected, but not totally disconnected, or connected (in the Euclidean sense), but not net-connected. 
On certain families of planar patterns and fractals

Moreover, an analogon of fractal percolation in the unit cube (see, e.g., $[27,18,17]$ ), called net percolation, has been introduced, and a sufficient condition for net percolation was proven [8].

The results obtained for limit net sets provide methods for the construction of random fractals with a certain type of "well distributed" structure (holes) by using net patterns/net matrices, but also for constructing percolating fractal sets and sets that have certain connectedness properties.

Later on this idea of identifying families of patterns according to their shape was used [10] in the study of the generalised Sierpiński carpets to which the next section is dedicated.

\subsection{Generalised Sierpiński carpets}

Generalized Sierpinski carpets are planar sets in the unit square that were introduced and studied in $[9,10]$. These sets generalise the Sierpiński carpets mentioned in the introduction. They differ in several aspects from a Sierpiński carpet defined as above: on the one hand, instead of using a single generating pattern, here we use a sequence of patterns in order to construct the generalised carpet, on the other hand, at any step $k$ of the construction, a $m_{k} \times m_{k}$ pattern is used, where $m_{k} \geq 2$, for all $k \geq 1$, and, moreover, at any two steps $k_{1} \neq k_{2}$ we may have distinct patterns, with $m_{k_{1}} \neq m_{k_{2}}$. Thus, generalised Sierpiński carpets are in general not self-similar. With the notations from Section 1.2 we introduce the following notions.

Let $\left\{\mathcal{A}_{k}\right\}_{k=1}^{\infty}$ be a sequence of non-empty patterns and $\left\{m_{k}\right\}_{k=1}^{\infty}$ be the corresponding width-sequence, i.e., for all $k \geq 1$ we have $\mathcal{A}_{k} \subseteq \mathcal{S}_{m_{k}}$. We let $\mathcal{W}_{1}=\mathcal{A}_{1}$, and call it the set of white squares of level 1 . For $n \geq 2$ we define the set of white squares of level $n$ by

$$
\mathcal{W}_{n}=\bigcup_{W \in \mathcal{A}_{n}, W_{n-1} \in \mathcal{W}_{n-1}}\left\{P_{W_{n-1}}(W)\right\}
$$

By defining three types of graphs associated to the patterns that generate the generalised Sierpiński carpets, necessary and sufficient conditions for the connectedness (with respect to the usual topology of the Euclidean plane) of these objects were proven [9].

A different approach, namely identifying certain families of patterns, was used [10] in order to study the structure of the sets obtained at the $n$th iteration in the construction of a generalised carpet, for $n \geq 1$, and it was shown that certain families of patterns provide total disconnectedness of the resulting fractals. Moreover, analogous results hold even in a more general setting [10]. This approach of the carpets provides the possibility 


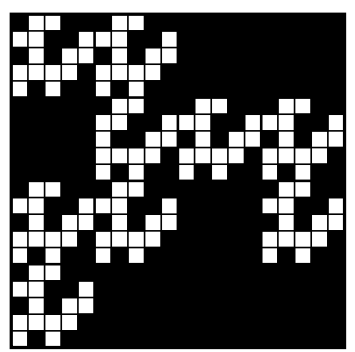

Figure 4: The set $\mathcal{W}_{2}$, constructed based on the patterns $\mathcal{A}_{1}$ and $\mathcal{A}_{2}$ shown in Figure 1, that can also be viewed as a 20-pattern

to construct disconnected carpets of box-counting dimension less than or even equal to 2 , as it is shown in an example in the more extended arXiv-version [11] of the published paper [10].

The results on connected generalised carpets [9] and on distances between points on the "classical" $s$-dimensional carpet [7] were extended in more recent work by Hoffmann [23]: analogues of the generalised Sierpiński carpets mentioned above, called generalised Sierpinski hypercubes, were defined and studied, and it has been shown that these sets are uniformly regular, i.e., the geodesic metric is comparable to the Euclidean metric. We also mention that in previous work, several authors $[2,6,24,31,34]$ studied objects called general Sierpinski carpets with respect to dimension and Hausdorff measure using rectangles instead of squares in the definition. While some of these carpets are self-affine, those defined by Barański [2] are not self-affine.

More recently, geometrical and topological properties of fractal squares, which are self-similar Sierpiński carpets as defined in the introduction, were studied [25, 39]. We note that the self-similar version of the limit net sets mentioned in Section 1.3 and the labyrinth fractals mentioned in Section 1.5 are fractal squares. Lau, Luo, and Rao [25] study the topological structure of a fractal square by studying the connected components. Moreover, recently there is considerable interest to study the Lipschitz equivalence of Cantor sets and of totally disconnected fractal squares, e.g., [26, 38].

In more recent work [14] dedicated to mixed labyrinth fractals, Steinsky and Cristea gave an other sufficient condition for the total disconnectedness of certain classes of generalised Sierpiński carpets, that occur in relation labyrinth fractals that we present in Section 1.5.

We note the combinatorics-flavoured approach of carpets by identifying special families among the generating patterns that provide certain properties, which was inspired by 
the results that were obtained for limit net sets, that is different from the approaches of other authors who have studied similar objects. On the other hand, Cristea and Steinsky used graphs a lot as a tool in order to characterise some properties of the patterns or of the prefractals obtained at some (finite) steps of the iterative construction of generalised Sierpiński carpets [9] or other carpets [12, 13, 14], and graphs were also used by other authors, e.g., when dealing with fractal squares [25].

\subsection{Labyrinth fractals}

An other new family of (self-similar) fractals, called labyrinth fractals, were introduced and studied during the last decade $[12,13]$. These fractal objects are (self-similar) dendrites and a special case of the Sierpiński carpets mentioned at the beginning of Section 1.1. First, self similar labyrinth fractals generated by a $4 \times 4$ labyrinth pattern were studied [12]. Originally, such a generator was called "labyrinth set" [12], not "labyrinth pattern", as used in later work on mixed labyrinth fractals $[14,15]$. Subsequently, by proving several quite technical lemmas and theorems, the results were extended [13] to the case of self-similar labyrinth fractals generated by $m \times m$ labyrinth patterns, for any $m \geq 5$.
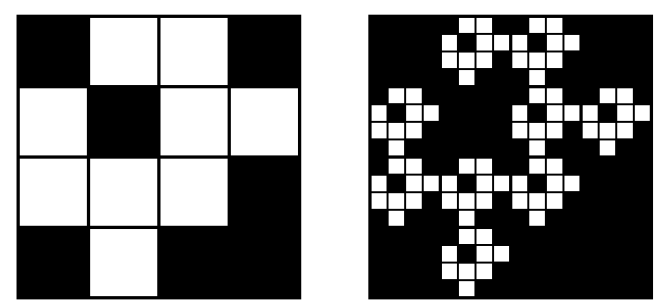

Figure 5: A $4 \times 4$ labyrinth pattern and the corresponding labyrinth set of level 2 (that can also be viewed as a $16 \times 16$ labyrinth pattern)

To the $m \times m$ pattern that generates a labyrinth fractal we associate the set of $\mathcal{W}_{1}$ of its (closed) white squares and call it the set of white squares of level 1 or labyrinth set of level 1, and we define $L_{1}=\cup_{W \in \mathcal{W}_{1}}$. By the iterative construction described in Section 1.1 we then obtain the sequence $\left\{\mathcal{W}_{n}\right\}_{n \geq 1}$, with $\mathcal{W}_{n} \subset \mathcal{S}_{m^{n}}$, and the decreasing sequence of compact sets $\left\{L_{n}\right\}_{n \geq 1}$.

A top exit in $\mathcal{W}_{n}$ is a white square in the top row of $\mathcal{W}_{n}$, such that there is also a white square in the same column in the bottom row. The bottom exit, left exit, and right exit are defined analogously. 
On certain families of planar patterns and fractals

A non-empty $m$-pattern $\mathcal{A} \subseteq \mathcal{S}_{m}, m \geq 3$ is called a $m \times m$-labyrinth pattern (in short, labyrinth pattern) if $\mathcal{A}$ satisfies satisfies the following three properties:

(1) $\mathcal{G}\left(\mathcal{W}_{n}\right)$ is a tree;

(2) exactly one top exit in $\mathcal{W}_{n}$ lies in the top row (of order $n$ ), exactly one bottom exit lies in the bottom row, exactly one left exit lies in the left column, and exactly one right exit lies in the right column;

(3) if there is a white square in $\mathcal{W}_{n}$ at a corner of $\mathcal{W}_{n}$, then there is no white square in $\mathcal{W}_{n}$ at the diagonally opposite corner of $\mathcal{W}_{n}$.

We note that the graph $\mathcal{G}(\mathcal{A})$ introduced in Section 1.2 can also be defined in the case $\mathcal{A}=\mathcal{W}_{n}, n \geq 1$. These graphs play an important role throughout the study of labyrinth sets and labyrinth fractals.

For any labyrinth pattern $\mathcal{A}$ and any integer $n \geq 1$, the labyrinth set (of level $n$ ) $\mathcal{W}_{n}$ has the above properties (1) - (3) of a labyrinth pattern [12].

The limit set $L_{\infty}$ of the decreasing sequence of compact sets $\left\{L_{n}\right\}_{n \geq 1}$ is called a labyrinth fractal. Every labyrinth fractal has four exits. The top exit of $L_{\infty}$ lies on the top edge of the unit square and is the intersection (point) $\cap_{n=1}^{\infty} T_{n}$, where $T_{n}$ is the top exit in $\mathcal{W}_{n}$, for all $n \geq 1$. The bottom exit, the left and the right exit of $L_{\infty}$ are defined analogously and lie correspondingly on the other edges of the unit square.

We say that an $m \times m$-labyrinth pattern $\mathcal{A}$ is horizontally blocked if the row (of squares) from the left to the right exit of $\mathcal{A}$ contains at least one black square, and it is called vertically blocked if the column (of squares) from the top to the bottom exit contains at least one black square. We remark that in Figure 1 the first two labyrinth patterns are both horizontally and vertically blocked, and the third pattern is neither horizontally, nor vertically blocked, while the $4 \times 4$ pattern shown in Figure 5 is both vertically and horizontally blocked. For more examples we refer to [12, 13, 14, 15].

Both topological and geometrical properties and aspects of the labyrinth sets and fractals were studied. It was proven that any self-similar labyrinth fractal $L_{\infty}$ is a dendrite, i.e., a locally connected continuum that contains no simple closed curve $[12,13]$.

Subsequently, the $\operatorname{arcs}$ in $L_{\infty}$ that connect exits of the fractal were studied, with emphasis on their length. In order to obtain results for the lengths of such arcs, we studied the lengths of paths in the tree $\mathcal{G}\left(\mathcal{W}_{n}\right)$ between exits in $\mathcal{W}_{n}$. Therefore, the path matrix $M$ of the labyrinth set $\mathcal{W}_{1}$ was introduced, which is a $6 \times 6$ matrix where each entry represents the number of a certain type of squares in one of the 6 paths in $\mathcal{G}\left(\mathcal{W}_{1}\right)$ between two exits of $\mathcal{W}_{1}$. (For more details on the possible 6 types of squares in a path in $\mathcal{G}\left(\mathcal{W}_{1}\right)$ see, e.g., [12].) The path matrix plays an essential role and is a powerful instrument when dealing with lengths of paths in $\mathcal{G}\left(\mathcal{W}_{n}\right)$ and with lengths of arcs between exits in $L_{\infty}$. Moreover, this matrix actually is the matrix of a substitution. 
On certain families of planar patterns and fractals

In order to prove the obtained results, several known theorems from different areas of mathematics were used: the Perron-Frobenius Theorem, the Hahn-MazurkiewiczSierpiński Theorem (that characterises local connectedness), the Jordan Curve Theorem, and a labyrinth version of the Steinhaus Chessboard Theorem, proven in [12].

It was essential to establish $[12,13]$ a recursion and prove that the $n$-th power $M^{n}$ of the path matrix gives information about the lengths of paths in $\mathcal{G}\left(\mathcal{W}_{n}\right)$. Moreover, it was shown that the path matrix of a $m \times m$ - labyrinth pattern (or set) is primitive if and only if the pattern (set) is horizontally and vertically blocked. Then, the PerronFrobenius Theorem for primitive matrices was used, see e.g. [40, Theorem 1.1, p.3], in order to obtain the asymptotics of the path lengths in $\mathcal{G}\left(\mathcal{W}_{n}\right)$ as $n$ tends to infinity. This subsequently lead to results about the lengths of arcs in the labyrinth fractals generated by both horizontally and vertically blocked labyrinth patterns. In the case of $m \times m$ patterns with $m \geq 5$, not just the path matrix mentioned above, but also use a second matrix, the reduced path matrix, was used in order to prove the infinite length of arcs in the fractal [13].

The main results on labyrinth fractals, both in the case when the fractal is generated by a $4 \times 4$ pattern and in the case when the generating pattern is $m \times m$, with $m \geq 5$, are contained in the following theorem $[12,13]$.

Theorem. If $L_{\infty}$ is the labyrinth fractal generated by a horizontally and vertically blocked $m \times m$-labyrinth pattern $(m \geq 4)$ with path matrix $M$, and $r$ is the spectral radius of $M$, then between any two points in $L_{\infty}$ there is a unique arc $\boldsymbol{a}$, the length of $\boldsymbol{a}$ is infinite, and the set of all points, at which no tangent to $\boldsymbol{a}$ exists, is dense in $\boldsymbol{a}$. Moreover, if $\boldsymbol{a}$ is an arc between two distinct points in $L_{\infty}$ then its box-counting dimension is $\operatorname{dim}_{B}(\boldsymbol{a})=\frac{\log (r)}{\log (m)}$.

The case when the labyrinth fractal is generated by a $4 \times 4$-pattern that is blocked only in one direction (e.g., only horizontally, but not vertically blocked) is also interesting: we have proven that then there exist both arcs of finite length and arcs of infinite length in the fractal. Moreover, in this case the box-counting dimension of every arc is 1 , while in the case of a labyrinth fractal generated by a both horizontally and vertically blocked pattern the dimension of such arcs is always strictly greater than 1 . For more details we refer to [12].

Mixed labyrinth fractals were defined and studied later [14], as a generalisation of the self-similar labyrinth fractals mentioned above. Here, the construction is analogous to that of the generalised Sierpiński carpets mentioned in Section 1.4, with the difference that all patterns that occur throughout the construction are labyrinth patterns. In other words, mixed labyrinth fractals are a special case of generalised Sierpiński carpets. As 
On certain families of planar patterns and fractals

an example, Figure 4 shows the labyrinth set of level $2, \mathcal{W}_{2}$, generated by the patterns $\mathcal{A}_{1}$ and $\mathcal{A}_{2}$ from Figure 1 .

Mixed labyrinth fractals are, like the self-similar labyrinth fractals mentioned above, dendrites [14], but here things get more complicated when studying lengths of paths in the graphs of mixed labyrinth sets of some level, and a lot more complicated when studying lengths of arcs in the fractal. The methods used in the self-similar case, based on the path matrix, can only be applied up to a certain point in the reasoning. This is due to the fact that in the self similar case the path matrix of a labyrinth set of level $n$ is just $M^{n}$, where $M$ is the path matrix of the generating pattern, while in the mixed case it is $M_{1} \cdot M_{2} \cdot M_{n}$, where $M_{k}$ is the path matrix of the pattern $\mathcal{A}_{k}$, for $k=1, \ldots, n$. Since there are no restrictions regarding the labyrinth patterns that occur in the generating sequence $\left\{\mathcal{A}_{k}\right\}_{k \geq 1}$, the methods used in the self-similar case in order to establish results on the asymptotical behavior of the path matrix associated to the labyrinth set of level $n$, for $n \rightarrow \infty$, cannot be applied here anymore, as soon as the sequence contains more than one pattern (unless the sequence is periodic, which is in general not the case). Moreover, there are also other properties that get lost when we give up self-similarity, e.g., in the case of mixed labyrinth fractals it is possible that an exit of the fractal lies, for some $n \geq 1$, in more than one white square of level $n$ of $\mathcal{W}_{n}$, while in the self-similar case each exit lies in a unique such white square of level $n$.

In a recently published paper [15] in was shown, that in the case of a mixed labyrinth fractal the theorem stated above does not hold. More precisely, one can prove the following two results.

Theorem. There exist sequences $\left\{\mathcal{A}_{k}\right\}_{k=1}^{\infty}$ of (both horizontally and vertically) blocked labyrinth patterns, such that the limit set $L_{\infty}$ has the property that for any two points in $L_{\infty}$ the length of the arc $a \subset L_{\infty}$ that connects them is finite. For almost all points $x_{0} \in a$ (with respect to the length) there exists the tangent at $x_{0}$ to the arc $a$.

Proposition. There exist sequences $\left\{\mathcal{A}_{k}\right\}_{k=1}^{\infty}$ of (both horizontally and vertically) blocked labyrinth patterns, such that the limit set $L_{\infty}$ has the property that for any two points in $L_{\infty}$ the length of the arc $a \subset L_{\infty}$ that connects them is infinite.

These results were proven by using a special family of labyrinth patterns, which we called "special cross patterns". An example of such a pattern is shown in Figure 6.

Here, the idea was to approximate the arcs that connect exits in the labyrinth fractal by special curves, which are related to the patterns used in the construction. For more details we refer to the paper [15]. Analogously to the self-similar case, by chosing suitable labyrinth patterns that are blocked in only one direction (e.g., horizontally but not vertically blocked), one can construct also in the mixed case labyrinth fractals where both arcs of finite length and arcs of infinite length exist between their points. 


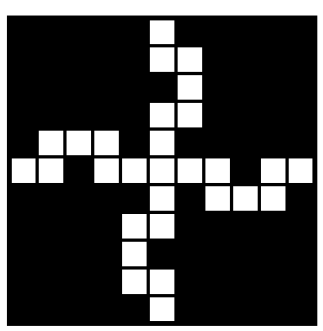

Figure 6: An example: a special cross pattern with width 11
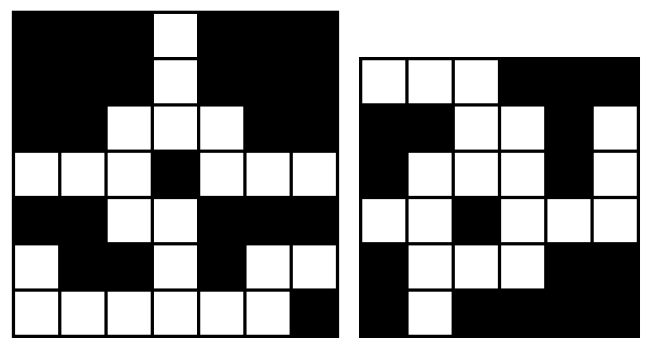

Figure 7: Examples: two wild labyrinth patterns, both vertically and horizontally blocked

The following conjecture was formulated [15]:

Conjecture. A sequence of both horizontally and vertically blocked labyrinth patterns with the property that the sequence of widths $\left\{m_{k}\right\}_{k \geq 1}$ is bounded, generates a mixed labyrinth fractal with the property that for any $x, y \in L_{\infty}$ the length of the arc in the fractal that connects $x$ and $y$ is infinite.

Finally, let us mention that in [14] it is also shown how, by relaxing the conditions imposed on the labyrinth patterns in order to construct wild labyrinth patterns and, correspondingly, (self-similar or mixed) wild labyrinth fractals, several properties of the labyrinth sets and fractals change, and in general the path matrices can not be used anymore in order to provide reliable information and results about the paths in the graphs of the labyrinth sets $\mathcal{G}\left(\mathcal{W}_{n}\right)$, for $n \geq 2$ or the lengths of arcs in the fractal. Figure 7 shows two wild labyrinth patterns. The first one has more than one horizontal exit pair, and the graph of the second one is only connected, but not a tree. Moreover, the connected net patterns mentioned in Section 1.3 are in particular $4 \times 4$ wild labyrinth patterns. 
On certain families of planar patterns and fractals

\subsection{Conclusions}

Although the fractals presented in this paper, limit net sets, generalised Sierpiński carpets and labyrinth fractals, can also be studied by using other approaches than the one used here, e.g., under the framework of IFS, graph-directed constructions, GDMS, random fractals or $V$-variable fractals, here we chose this unifying and rather combinatorial approach based on planar patterns. The motivation of this fact is that, in our opinion, this is a way to bring them closer to other sciences, to specialists from other fields, to a wider audience in general, since the notion of "pattern" is very intuitive, wide-spread (even in every day life) and somehow basic for the understanding. Moreover, as it follows from the cited papers, patterns are sufficient in order to construct and study these new, special families of fractals with remarkable properties.

The idea is that in this approach mainly by identifying families of patterns or a few properties of the patterns that are easy to check, one can generate fractals of prefractals with desired topological or geometrical properties (like types or degrees of connectedness, or lengths of arcs between points in the fractal), or, in addition, with desired fractal dimension.

It is important to add here that physicists use fractals like those mentioned above as models in different areas, e.g, for the study of materials or of diffusion in porous matter [20, 42, 36], planar nanostructures [22], or even for the construction of new, more performant devices (e.g. radar antennas) [37].

In this context it is worth to remark that, while the mathematicians focus mainly on the fractal, i.e., on the objects obtained as the limit of the iterative construction, the physicists are usually more interested in some prefractal obtained after a high enough number of iterations. In other words, while the mathematicians are mainly interested in what happens in the infinite, in the limit, the physicists are interested in what is obtained at a finite step that approximates the limit well enough, or where the scale is fine enough, but not infinitely fine. Let us give an example. From the point of view of research in physics, the properties (1) and (2) of labyrinth patterns are essential, and they are sufficient, since the property (3) of labyrinth patterns only plays a role in the limit, when dealing with the resulting labyrinth fractal: the fact that the resulting labyrinth fractal is a dendrite played an essential role when proving some of the other results, e.g., those on the length of arcs in the fractal.

Finally, let us mention that due to the interesting properties of limit net sets, generalised Sierpiński carpets and labyrinth fractals, at the moment this research is continued on new families of fractals, that are further generalisations of the objects mentioned here: there is a lot of magic and still a lot to discover in this field. 


\subsection{Acknowledgment}

The author is supported by the Austrian Science Fund (FWF), Project P27050-N26, and by the Austrian Science Fund (FWF) Project F5508-N26, which is part of the Special Research Program "Quasi-Monte Carlo Methods: Theory and Applications".

\section{References}

[1] C. Bandt, M. Mubarak, Distribution of distances and interior distances for certain self-similar measures, The Arabian Journal for Science and Engineering, 29 (2004), No. $2 \mathrm{C}$

[2] K. Barański, Hausdorff dimension of the limit sets of some planar geometric constructions, Adv. Math., 210 (2007), No. 1, 215-245

[3] M.F. Barnsley, Fractals Everywhere, Academic Press, Boston, 1984

[4] M. F. Barnsley, SuperFractals, Cambridge University Press, New York, 2006

[5] M. T. Barlow, E. A. Perkins, Brownian motion on the Sierpinsski gasket, Probability Theory and Related Fields, 79, 543-623, 1988

[6] T. Bedford, Crinkly curves, Markov partitions and box dimension in self-similar sets, Ph.D. Thesis (1984), University of Warwick

[7] L. L. Cristea, A geometric property of the Sierpiński carpet Quaestiones Mathematicae, 28 (2005), No. 2, 251-262

[8] L. L. Cristea, On the connectedness of limit net sets, Topology and its Applications 155 (2008), No. 16, 1808-1819

[9] L. L. Cristea, B. Steinsky, Connected generalised Sierpinski carpets, Topology and its Applications 157 (2010), 1157-1162

[10] L. L. Cristea, B. Steinsky, On totally disconnected generalised Sierpiński carpets, Bulletin Mathematique de la Societe des Sciences Mathematiques de Roumanie, Tome 57 (105) (2014), No. 1 27-34

[11] L. L. Cristea, B. Steinsky, On totally disconnected generalised Sierpinsski carpets, arXiv:1303.4883v1[math.GN] 
[12] L. L. Cristea, B. Steinsky, Curves of infinite length in $4 \times 4$-labyrinth fractals, Geometriae Dedicata, Vol. 141, Issue 1 (2009), 1-17

[13] L. L. Cristea, B. Steinsky, Curves of infinite length in labyrinth fractals, Proceedings of the Edinburgh Mathematical Society Volume 54, Issue 02 (2011), 329-344

[14] L. L. Cristea, B. Steinsky, Mixed labyrinth fractals, article in press: Topol. Appl. (2017), http://dx.doi.org/10.1016/j.topol2017.06.022

[15] L.L. Cristea, G. Leobacher On the length of arcs in mixed labyrinth fractals, Monatsh Math (2017). doi:10.1007/s00605-017-1056-8

[16] M. L. Curtis, M. K. Fort Jr., Singular homology of one-dimensional spaces, Ann. of Math. (2), 69 (1959), 309-313

[17] K. J. Falconer, Random Fractals, Math. Proc. Camb. Phil. Soc. 100 (1986), 559582

[18] K. J. Falconer, Fractal Geometry. Mathematical Foundations and Applications, John Wiley \& Sons, Chichester 1990

[19] K. J. Falconer, Techniques in Fractal Geometry. Mathematical Foundations and Applications, John Wiley \& Sons, Chichester 1997

[20] A. Franz, C. Schulzky, S. Tarafdar, K. Hoffmann, The pore structure of Sierpiński carpets, Journal of Physics. A. Mathematical and General, 34 (2001), No. 42, 87518765

[21] U. Freiberg, B. M. Hambly, J.E. Hutchinson, Spectral Asymptotics for V-variable Sierpinski Gaskets, arXiv:1502.00711 [math.PR]

[22] V. I. Grachev, A. A. Potapov, V. A. German, Fractal Labyrinths and Planar Nanostructures, PIERS Proceedings, Stockholm, Sweden, Aug. 12-15, 2013

[23] H. Hoffmann, A metric property of Sierpinski hypercubes, Quaest. Math, 36 (2013), $197-208$

[24] S. P. Lalley, D. Gatzouras, Hausdorff and box dimensions of certain self-affine fractals, Indiana Univ. Math. J., 41 (1992), No. 2, 533-568

[25] K.-S. Lau, J. J. Luo, H. Rao, Topological structure of fractal squares, Mathematical proceedings of the Cambridge Philosophical Society, Vol. 155 (2013), Issue 01, 7386 
[26] K.-S. Lau, J. J. Luo, On the classification of fractal squares, Fractals 24 (2016), No. 01, DOI: http://dx.doi.org/10.1142/S0218348X16500080

[27] B. Mandelbrot, The Fractal Geometry of Nature, W.H. Freeman \& Co., San Francisco, 1983

[28] R. D. Mauldin, S.C. Williams, Random constructions: asymptotic geometric and topological properties, Trans. Amer. Math. Soc. 295 (1986), 325-346

[29] R. D. Mauldin, S. C. Williams, Hausdorff dimension in graph directed constructions, Trans. Amer. Math. Soc. 309 (2) (1988), 811-829

[30] R. D. Mauldin, M. Urbański, Graph Directed Markov Systems: geometry and dynamics of limit sets, Cambridge Tracts in Mathematics, Cambridge University Press, 2003

[31] C. McMullen The Hausdorff dimension of general Sierpiński carpets, Nagoya Math. J., 96 (1984), 1-9

[32] P. A. P. Moran, Additive functions of intervals and Hausdorff measure, Proceedings of the Cambridge Philosophical Society 42 (1946), 15-23

[33] H. Niederreiter, Point sets and sequences with small discrepancy, Mh. Math. 104 (1987), 273-337

[34] Y. Peres, The self-affine carpets of McMullen and Bedford have infinite Hausdorff measure, Math. Proc. Cambridge Philos. Soc. 116 (1994), No. 3, 513-526

[35] Y. Pesin, H. Weiss, On the Dimension of Deterministic and Random Cantor-like Sets. Symbolic Dynamics and the Eckmann-Ruelle Conjecture, Commun. Math. Phys. 182 (1996), 105-153

[36] A. A. Potapov, V.I. Grachev, Fractal Labyrinths: Physics and Fractional Operators, PIERS Proceedings, Moscow, August 19-23, 2012

[37] A. A. Potapov, W. Zhang, Simulation of New Ultra-Wide Band Fractal Antennas Based on Fractal Labyrinths, Proceedings of the 2016 CIE International Conference on Radar, Guangzhou, Oct 10-12, 2016, 319-323

[38] H Rao, H.-J. Ruan, L.-F. Xi, Lipschitz equivalence of self-similar sets, C. R. Math. Acad. Sci. Paris, 342 (3) (2006), 191-196

[39] K. A. Roinestad, Geometry of fractal squares, Ph. D. Thesis (2010), The Virginia Polytechnic Institute and State University 
[40] E. Seneta, Non-negative matrices and Markov chains, 2nd, edn, Springer, 2006

[41] W. Sierpiński, Sur une courbe cantorienne qui contient une image biunivoque et continue de toute courbe donnée, C.R. Acad. Sci. Paris, 162 (1916), 629-632

[42] S. Tarafdar, A. Franz, C. Schulzky, K. H. Hoffmann, Modelling porous structures by repeated Sierpinski carpets, Physica A 292 (2001), 1-8

[43] G. T. Whyburn, Topological characterization of the Sierpinski curve, Fund. Math. 45 (1958), 320-324 
